HRJ

V. 2 n.10 (2021)

Recebido: $29 / 11 / 2020$

Aceito: 20/02/2021

\title{
Perfil de infecções em artroplastia de quadril: uma revisão integrativa
}

\author{
Thiago Filipe Novato ${ }^{1}$ \\ Mirce Meire Gonçalves de Sousa Wilk ${ }^{2}$ \\ Luciene Teixeira Araújo ${ }^{3}$ \\ Edivalda Pereira de Abreu ${ }^{4}$
}

1. Enfermeiro, Especialista em Centro Cirúrgico pelo Programa de Residência em Enfermagem em Centro Cirúrgico da ESCS/FEPECS/SES - DF Brasília (DF), Brasil. Lattes: http://lattes.cnpq.br/9736021075426503

2. Enfermeira da Secretaria de Saúde do Distrito Federal, Preceptora do Programa de Residência Uniprofissional de Enfermagem em Centro Cirúrgico - FEPECS/SES-DF. Pós-graduanda em Mestrado Acadêmico em Ciências da Saúde pela Fundação de Ensino e Pesquisa em Ciências da Saúde (FEPECS). Lattes: http://lattes.cnpq.br/9478783037542964

3. Enfermeira da Secretaria de Saúde do Distrito Federal, Preceptora do Programa de Residência Uniprofissional de Enfermagem em Centro Cirúrgico - FEPECS/SES-DF. Especialista em Controle de Infecção Hospitalar. Lattes: http://lattes.cnpq.br/4836270279928946

4. Enfermeira da Secretaria de Saúde do Distrito Federal, Preceptora do Programa de Residência Uniprofissional de Enfermagem em Centro Cirúrgico - FEPECS/SES-DF. Especialista em Terapia Intensiva, Mestre em Ciências Ambientais e Saúde e Doutora em Psicologia. Lattes: http://lattes.cnpq.br/4217797200699610

E-mail para correspondência: filipe.thiago@yahoo.com.br

\section{RESUMO}

INTRODUÇÃO: Procedimentos cirúrgicos são realizados em grande quantidade em todo o mundo, sendo assim, os riscos de infecções aumentam, o que gera divergência no tratamento, prolongamento da internação, custos, entre outros. A artroplastia de quadril é uma cirurgia de grande porte, que se dispõe hoje em dia de novos materiais e técnicas para a realização de um procedimento mais seguro, e tornarem-se menores as taxas de infecções. Contudo, toda cirurgia tem a probabilidade de desenvolver infecções, e quando se reconhece o agente etiológico, o tratamento é mais direcionado e conciso. OBJETIVO: Analisar o perfil de infecções cirúrgicas em artroplastia de quadril. MÉTODOS: Trata-se de uma revisão integrativa, de estudos disponibilizados na plataforma da Biblioteca Virtual de Saúde (BVS), com base de dados LILACS, PUBMED e SCIELO, com os descritores: Infecção, Artroplastia e Quadril. Os artigos abordados nos resultados são de publicações referentes aos anos de 2015 a 2020, conforme os critérios de inclusão e exclusão. RESULTADOS: As amostras relacionadas à temática do estudo evidenciaram Hipertensão Arterial, Diabetes Mellitus e Tabagismo como os fatores de risco mais associados aos pacientes submetidos a artroplastia de quadril. Como escolha para antibioticoprofilaxia foi utilizada cefazolina na maioria dos casos e Staphylococcus aureus foi o agente mais encontrado nas culturas. CONCLUSÕES: Conclui-se que é de alta relevância manter os cuidados no pós-operatório e caso apresente sinais de infecção, a cultura precoce favorece o tratamento.

Palavras-chaves: Infecção; Artroplastia; Quadril 


\title{
Profile of infections in hip arthroplasty: an integrative review
}

\begin{abstract}
INTRODUCTION: Surgical procedures are performed in large quantities all over the world, thus, the risks of infections increase, which generates divergence in treatment, prolongation of hospitalization, costs among others. Hip arthroplasty is a major surgery, which nowadays has new materials and techniques to carry out a safer procedure, and to make infection rates lower. However, every surgery is likely to develop infections, and when the etiologic agent is recognized, treatment is more targeted and concise. OBJECTIVE: The aim of the study is to analyze the profile of surgical infections in hip arthroplasty. METHODS: It is an integrative review of studies available on the Virtual Health Library (VHL) platform, using LILACS, PUBMED and SCIELO databases, with the descriptors; Infection, Arthroplasty and Hip. The articles covered in the results are from publications referring to the years 2015 to 2020, according to the inclusion and exclusion criteria. RESULTS: Samples related to the theme of the study showed Arterial Hypertension, Diabetes Mellitus and Smoking as the risk factors most associated with patients undergoing hip arthroplasty, as the choice for antibiotic prophylaxis, cefazoline was used in most cases and Staphylococcus aureus as the most commonly found agent in cultures. CONCLUSIONS: We conclude that it is highly relevant to maintain care in the postoperative period and in case of signs of infection, early culture favors treatment.
\end{abstract}

Key words: Infection; Arthroplasty; Hip. 


\section{INTRODUÇÃO}

Nos dias atuais, os pacientes hospitalizados, que são submetidos a procedimento invasivo, apresentam $10 \%$ de chance de desenvolver infecção ${ }^{1}$. Existem critérios para definir se é infecção hospitalar, tais como o tempo da manifestação dos sintomas e a data da admissão ${ }^{1 .}$

Nos serviços de Saúde do Brasil, as infecções em sítio cirúrgico (ISC) mostram divergências relacionadas com a segurança do paciente, principalmente as infecções que se manifestam em pacientes que são submetidos a cirurgias com ou sem implantes, acometendo qualquer tecido, e que se manifestam até o $30^{\circ}$ dia de pós-operatório, e algumas até um ano se houver implante. $^{2}$

Em National Healthcare Safety Network (NHSN) foi realizado um estudo de 850 mil cirurgias gerais realizadas nos EUA, e mostrou o número novo de casos igual a $1,9^{\mathrm{a}} \%$, sendo que no Brasil, os novos casos de ISC em cirurgias variam de $1,4 \%$ a $38,8 \%{ }^{3}$

A incidência da ISC depende de vários fatores, que envolvem condições do paciente, virulência do microrganismo, grau de contaminação, entre outros. ${ }^{4}$

Nos dias de hoje, a resistência bacteriana tem se tornado uma questão muito delicada em se tratando de saúde, e por isso, tende-se o aumento gradativo na morbidade e mortalidade dos pacientes. $^{5}$

Nos últimos tempos, a resistência bacteriana tem causado, tanto nos ambientes hospitalares quanto na comunidade, uma grande divergência, e muitos patógenos são resistentes a quase todos antimicrobianos, o que causa um temor nas redes hospitalares. ${ }^{6}$

A resistência tem crescido bastante nos últimos tempos, o que faz com que o conhecimento do perfil de sensibilidade das bactérias que mais acometem os pacientes seja estudado. $^{7}$ 
O uso correto de antimicrobianos é muito importante, pois o uso indiscriminado e inadequado faz com que se tenha o desenvolvimento de cepas resistentes. ${ }^{7}$

Estudos mostraram que entre 1990 e 1996 nos EUA, o Staphylococcus aureus foi o agente causador mais comum das infecções hospitalares, isso, baseado no Sistema Nacional de Vigilância de Infecção Hospitalar dos EUA, o que causou a sua alta predominância em pneumonias e infecções cirúrgicas. ${ }^{6}$

Olhando para um amplo contexto relacionado a complicações pós-operatórias, a ISC se encontra como a principal, e partindo desse pressuposto, vemos a importância de se criarem estratégias tais como, acompanhamento pós a alta do paciente, sendo que 12 a $84 \%$ dessas infecções são diagnosticadas durante essa vigilância. ${ }^{2}$ E isso é importante para a obtenção de indicadores conclusivos para que se tenha uma diminuição das subnotificações, e os reais dados acerca da incidência da ISC. ${ }^{2}$

As infecções que podem ocorrer nas artroplastias de quadril, geralmente, são próximas de $1 \%$, mas é de suma importância a identificação dos fatores para que a prevenção e o tratamento dessas infecções sejam adequados. ${ }^{8}$

Se formos olhar os impactos que as infecções em sitio cirúrgico têm sobre o paciente e sobre a instituição, destacamos que envolve aumento de gastos em relação ao tratamento do paciente e o tempo aumentado que esses pacientes ficam internados. Os pacientes que desenvolvem infecções têm o risco aumentado de morte em relação aos outros pacientes que não desenvolvem. ${ }^{3}$

Diante do exposto, o presente trabalho foi norteado pela seguinte pergunta: qual o perfil das infecções em cirurgias de artroplastia de quadril?

Mesmo com novos protocolos, mudanças de hábitos, e até mesmo desenvolvimentos tecnológicos, precisamos aprimorar a assistência ao paciente e reconhecer o perfil microbiológico do hospital para um tratamento melhor e mais adequado ao paciente. 
Sendo assim o presente estudo tem como objetivo analisar, através de uma revisão integrativa, o perfil de infecções em artroplastia de quadril.

\section{MÉTODO}

O presente trabalho trata-se de uma revisão integrativa, que visa a importância de vários modelos de estudos práticos e teóricos, para uma efetiva discussão do tema proposto.

A revisão integrativa é um método bem especifico, que se baseia no resumo dos estudos realizados no passado, para que a partir disso seja fornecido um conhecimento mais amplo sobre algo especifico. Esse tipo de revisão se baseia em definir uma análise sobre o que já foi realizado e estudado em pesquisas anteriores acerca de um determinado assunto. ${ }^{9}$

A revisão integrativa é realizada por etapas; essas etapas foram desenvolvidas por 06 passos. Inicialmente, identifica-se o assunto proposto e realiza-se a seleção da questão de pesquisa, seguidos pelo segundo passo que é estabelecer os critérios que se adentram no objetivo, tais como inclusão e exclusão nas buscas. O terceiro passo trata do que será extraído dos estudos para a formação da análise; o quarto seria a parte de avaliação dos estudos que foram inclusos na pesquisa, seguido pelo quinto passo que é a interpretação dos resultados apresentados e finalizado pelo sexto passo que é a apresentação da revisão proposta. ${ }^{10}$

O período do levantamento dos dados foi realizado nos meses de agosto e setembro de 2020. As bases de dados pesquisadas foram: LILACS, PUBMED, SCIELO, disponíveis na BVS - Biblioteca Virtual em Saúde, utilizando os seguintes descritores: Artroplastia; Infecção; Quadril.

Foram analisados 60 artigos com textos completos, cujos critérios de inclusão foram: artigos entre 2015 e 2020, cirurgias ortopédicas e de artroplastia de quadril que desenvolveram ISC e artigos com descrição do perfil etiológico das infecções.

Os critérios de exclusão foram: artigos que não se enquadravam no objetivo da pesquisa, cirurgias além da artroplastia de quadril e artigos que não demonstravam perfil 
microbiológico. Abaixo segue o fluxograma referente aos artigos pesquisados e selecionados segundo os descritores e base de dados.

Fluxograma 1: Estratégia de busca nas bases de dados, período de 2015 a 2020

\begin{tabular}{|c|c|}
\hline Estratégia de Busca - BVS & \multirow{3}{*}{$\begin{array}{l}\text { Estudos } \\
\text { excluídos } \\
\text { por não } \\
\text { terem } \\
\text { texto } \\
\text { completo }\end{array}$} \\
\hline $\begin{array}{l}\text { - Lilacs, Pubmed, Scielo. } \\
\text { Total: } 1.166\end{array}$ & \\
\hline$\downarrow$ & \\
\hline $\begin{array}{l}\text { Estudos com potencial de } \\
\text { inclusão }\end{array}$ & № 560 \\
\hline № 60 & \\
\hline$\downarrow$ & $\begin{array}{l}\text { excluídos } \\
\text { por não }\end{array}$ \\
\hline $\begin{array}{l}\text { Estudos incluídos na } \\
\text { revisão }\end{array}$ & $\begin{array}{l}\text { atenderem } \\
\text { critérios } \\
\text { de } \\
\text { inclusão }\end{array}$ \\
\hline № 7 & № 599 \\
\hline
\end{tabular}

Fonte: Dados da pesquisa, 2020. 


\section{RESULTADOS E DISCUSSÃO}

\section{Tabela 1: Descrição dos artigos}

\begin{tabular}{|c|c|c|c|c|c|}
\hline $\mathbf{N}^{\mathbf{0}}$ & Título do artigo & Autor/Ano & Descritores & Objetivo & Evidências \\
\hline 01 & $\begin{array}{l}\text { Análise das infecções } \\
\text { de Sitio Cirúrgico em } \\
\text { pacientes pediátricos } \\
\text { após } \\
\text { ortopédica: Um estudo } \\
\text { caso-controle. }\end{array}$ & CHAGAS, et al, 2017 & Infecção & $\begin{array}{l}\text { Descrever taxa de infecção no sítio } \\
\text { cirúrgico em crianças submetidas à } \\
\text { cirurgia ortopédica em centro de } \\
\text { referência e analisar o perfil desses } \\
\text { pacientes. }\end{array}$ & $\begin{array}{l}\text { Das infecções apresentadas } 8 \text { foram superficiais e } 2 \text { de } \\
\text { órgão/cavidade. Desses 10, foram coletados materiais } \\
\text { biológicos de } 7 \text {, sendo que } 5 \text { pacientes tiveram } \\
\text { crescimento do agente, sendo } 4 \text { tendo a presença de } \\
\text { Staphyloccoccus aureus, sendo um deles resistente à } \\
\text { meticilina (MRSA), e } 1 \text { tendo apresentado Enterobacter } \\
\text { cloacae. }\end{array}$ \\
\hline 02 & $\begin{array}{l}\text { Artroplastia de quadril } \\
\text { em idosos } \\
\text { hospitalizados e uso de } \\
\text { antibioticoprofilaxia }\end{array}$ & $\begin{array}{l}\text { DULPAZ, } \\
\text { PAGNUSSAT, HAHN, } \\
2018\end{array}$ & $\begin{array}{l}\text { Artroplastia de } \\
\text { Quadril }\end{array}$ & $\begin{array}{l}\text { Descrever o perfil epidemiológico e } \\
\text { clínico de idosos hospitalizados } \\
\text { para artroplastia de quadril e } \\
\text { verificar a adesão aos protocolos de } \\
\text { antibioticoprofilaxia em cirurgias } \\
\text { de quadril, ressaltando a } \\
\text { importância do mesmo em } \\
\text { corroborar com as estatísticas } \\
\text { nacionais nessa população. }\end{array}$ & $\begin{array}{l}\text { Em relação à cirurgia e ao grau de contaminação foram } 57 \\
\text { cirurgias limpas, } 2 \text { potencialmente infectadas e } 1 \\
\text { infectada. } \\
\text { A prescrição de antibiótico pós procedimento ocorreu em } \\
97 \% \text {, o mais utilizado foi a cefazolina de } 1 \mathrm{~g} \text { de } 6 / 6 \mathrm{EV} \text { em } \\
73,4 \% \text { da amostra. O estudo mostrou que a taxa de ISC } \\
\text { foi de } 0,99 \% \text { e o microrganismo identificado foi } \\
\text { Pseudomonas. }\end{array}$ \\
\hline $\mathbf{0 3}$ & $\begin{array}{l}\text { Um protocolo para } \\
\text { artroplastia encenada } \\
\text { para salvar a não-união }\end{array}$ & $\begin{array}{l}\text { EBIED, A.M., } \\
\text { ELSEEDY, A.I. \& } \\
\text { GAMAL, O, } 2016\end{array}$ & $\begin{array}{l}\text { Artroplastia de } \\
\text { quadril, } \\
\text { Infecção }\end{array}$ & $\begin{array}{l}\text { Avaliar se um protocolo de } \\
\text { tratamento em etapas produz bons } \\
\text { resultados nesses casos difíceis. }\end{array}$ & $\begin{array}{l}\text { O artigo revela que foi encontrado em todos os pacientes, } \\
\text { pela aspiração, no pré-operatório em } 17 \text { casos, ou } \\
\text { amostras que foram coletadas na primeira etapa em } 9\end{array}$ \\
\hline
\end{tabular}




\begin{tabular}{|c|c|c|c|c|c|}
\hline & $\begin{array}{l}\text { infectada de fraturas de } \\
\text { quadril. }\end{array}$ & & & & $\begin{array}{l}\text { pacientes, o organismo infectante Estafilococus aureus, } \\
\text { resistente à meticilina ( MRSA ), tendo a ocorrência de } 13 \\
\text { e } 26 \text {, outros microrganismos foram encontrados tais como } \\
\text { Klebsiella e Epidermids resistente à meticilina (MRSE). }\end{array}$ \\
\hline 04 & $\begin{array}{l}\text { Infecção Cirúrgica em } \\
\text { pacientes submetidos a } \\
\text { cirurgia ortopédica com } \\
\text { implante. }\end{array}$ & $\begin{array}{l}\text { FRANCO, ERCOLE, } \\
\text { MATTIA, } 2015 .\end{array}$ & Infecção & 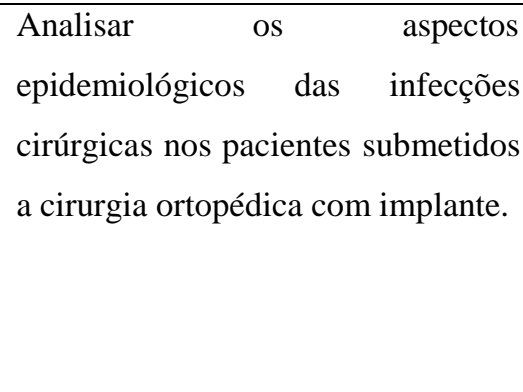 & $\begin{array}{l}\text { Das } 28 \text { infecções, } 26 \text { foram diagnosticadas com } 90 \text { dias de } \\
\text { pós-operatório. Teve ISC superficial de } 6,7 \text {, profunda de } \\
4,5 \text { e osteomielite de } 1,3 \text {. Em relação à incidência de ISC } \\
\text { em artroplastia de quadril, a redução aberta de fratura } \\
\text { contribuiu com } 12,8 \% \text { e de modo geral o agente com } \\
\text { maior predominância foi o Staphylococcus aureus }\end{array}$ \\
\hline 05 & $\begin{array}{l}\text { Caracterização de } \\
\text { artroplastias de quadril } \\
\text { e joelho e fatores } \\
\text { associados à infecção. }\end{array}$ & PINTO, et al, 2015 & $\begin{array}{l}\text { Artroplastia de } \\
\text { quadril, } \\
\text { infecção }\end{array}$ & $\begin{array}{l}\text { Caracterizar as artroplastias, } \\
\text { calcular a taxa de infecção cirúrgica } \\
\text { e identificar fatores de risco } \\
\text { relacionados. }\end{array}$ & $\begin{array}{l}\text { No período de estudo foram feitas } 421 \text { artroplastias totais } \\
\text { em } 346 \text { pacientes, sendo que } 213 \text { foram artroplastia total } \\
\text { de quadril. Foram } 18 \text { casos de infecção em } 18 \text { pacientes } \\
\text { que tiveram a taxa de prevalência de } 4,3 \% \text {. E nas } \\
\text { infecções de artroplastia total de quadril, a taxa de } \\
\text { prevalência foi de } 2,8 \% \text {, equivalente a } 6 \text { casos. Obteve-se } \\
\text { cultura de } 17 \text { pacientes, onde o agente com maior } \\
\text { predominância foi o Staphylococcus aureus. }\end{array}$ \\
\hline 06 & $\begin{array}{l}\text { Aspectos } \\
\text { epidemiológicos das } \\
\text { infecções de sitio } \\
\text { cirúrgico em cirurgias }\end{array}$ & MIRANDA, 2017 & Infecção & 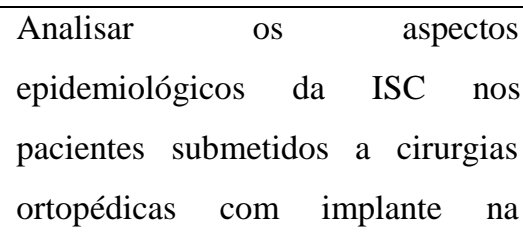 & $\begin{array}{l}\mathrm{Na} \text { amostra do estudo, as artroplastias de quadril } \\
\text { correspondiam a um número maior de procedimentos, } \\
\text { sendo 263. A maioria das cirurgias foi classificada como }\end{array}$ \\
\hline
\end{tabular}




\begin{tabular}{|c|c|c|c|c|c|}
\hline & $\begin{array}{l}\text { ortopédicas } \\
\text { implante em um } \\
\text { hospital de reabilitação }\end{array}$ & & & $\begin{array}{l}\text { unidade de Brasília da rede Sarah } \\
\text { Hospitais de Reabilitação. }\end{array}$ & $\begin{array}{l}\text { limpa com número de } 579 \text {, } 45 \text { como potencialmente } \\
\text { contaminadas e } 2 \text { contaminadas. } \\
21 \text { apresentaram ISC, sendo } 1 \text { paciente teve a ocorrência } \\
\text { de } 2 \text { cirurgias infectadas, tendo assim } 22 \text { cirurgias que } \\
\text { apresentaram ISC. Em } 22 \text { ISC, tiveram } 41 \text { amostras de } \\
\text { culturas, } 34 \text { tiveram culturas positivas, } 5 \text { negativas e } 2 \text { de } \\
\text { crescimento polimicrobiano. Foram isolados } 36 \text { agentes, } \\
\text { sendo o Staphylococcus aureus mais prevalente. }\end{array}$ \\
\hline
\end{tabular}

Fonte: Dados da pesquisa, 2020. 


\section{ARTROPLASTIA DE QUADRIL E PRINCIPAIS MICRORGANISMOS}

No primeiro artigo analisado (CHAGAS et al, 2017) foi realizado o levantamento das ISC em pacientes pediátricos, com ênfase em cirurgias ortopédicas. $\mathrm{O}$ trabalho foi feito na enfermaria pediátrica do Instituto Nacional de Traumatologia e Ortopedia Jamil Haddad (INTO), que é hospital do SUS, referência em tratamento de patologias osteoarticulares. Foram analisados alguns prontuários de pacientes que foram submetidos a cirurgias ortopédicas no período de 2 anos. No total foram utilizados 347 prontuários, sendo que 10 tiveram ISC, o que equivale a 2,88\%. Das infecções apresentadas 8 eram superficiais e 2 de órgão/cavidade. Desses 10, foram coletados materiais biológicos de 7, sendo que 5 pacientes tiveram crescimento do agente, sendo 4 tendo a presença de Staphyloccoccus aureus, em um deles resistente à meticilina (MRSA), e 1 tendo apresentado Enterobacter cloacae. ${ }^{11}$

No segundo estudo, DULPAZ et al, 2018, o artigo descreve o perfil epidemiológico e clínico de idosos hospitalizados para serem submetidos à artroplastia de quadril. Trata-se de um estudo transversal, retrospectivo, realizado em pacientes com idade superior/igual há 60 anos, que foram submetidos à cirurgia do quadril entre janeiro e dezembro de 2016. O estudo mostrou que a taxa de ISC foi de $0,99 \%$ e o microrganismo identificado foi Pseudomonas. ${ }^{12}$

O terceiro artigo (EBIED et al, 2016), trata-se de um estudo prospectivo de 27 pacientes que tiveram infecções profundas no quadril com tratamento falho de fratura de quadril. Foram tratados entre junho de 2007 e setembro de 2011. A idade média dos pacientes foi de 48,9 anos, na faixa de 26 a 74 anos de idade. A infecção foi controlada em todos os casos que completaram o protocolo de tratamento sem recidiva no último seguimento. $\mathrm{O}$ artigo revela que foi encontrado, em todos os pacientes pela aspiração no pré-operatório, em 17 casos, ou amostras que foram coletadas na primeira etapa em 9 pacientes, o organismo infectante Estafilococus aureus resistente à meticilina (MRSA), tendo a ocorrência de 13 e 
26; outros microrganismos encontrados foram Klebsiella e Epidermids resistente à meticilina (MRSE). ${ }^{13}$

No quarto artigo (FRANCO et al, 2015), foi realizado um estudo de coorte concorrente de 222 pacientes que foram submetidos a cirurgias ortopédicas com implantes em 2011, no período de maio a setembro. Todos os pacientes foram acompanhados por via telefônica. Os critérios de inclusão foram: idade maior que 16 anos, que possuíam telefone, e que tivessem ciência sobre os sinais de infecções, entre outros. Em relação à incidência de ISC em artroplastia de quadril, a redução aberta de fratura contribuiu com 12,8\% e de modo geral o agente com maior predominância foi o Staphylococcus aureus. ${ }^{14}$

O quinto artigo, de PINTO et al, 2015, é um estudo de coorte retrospectivo que foi realizado em um hospital de ensino da capital paranaense. O projeto aconteceu em 36 meses, de janeiro de 2010 a dezembro de 2012, que incluiu todos os procedimentos cirúrgicos de artroplastia de quadril e joelho. Obteve-se cultura de 17 pacientes, e o agente com maior predominância foi o Staphylococcus aureus. ${ }^{15}$

No sexto artigo (MIRANDA, 2017) foi realizado um estudo de coorte não concorrente de 517 pacientes que foram submetidos a 626 procedimentos cirúrgicos ortopédicos com implante na rede Sarah hospital de reabilitação em Brasília, no período de janeiro de 2010 e julho de 2015. Em 22 ISC, tiveram 41 amostras de culturas, 34 tiveram culturas positivas, 5 culturas negativas e 2 de crescimento polimicrobiano. Foram isolados 36 agentes, sendo o Staphylococcus aureus o mais prevalente. ${ }^{16}$

O sétimo artigo (CUNHA, 2019) trata-se de um estudo de coorte não concorrente, de 149 pacientes, que realizaram artroplastia de quadril e de joelho, durante o período de 2015 e maio de 2018 em um Hospital de Belo Horizonte. Das 6 ISC, houve coleta de 3 culturas, tendo assim o crescimento de Enterobacter cloacae em secreção da ferida, Acinetobacter baumannii e Stenotrophomonas maltophilia no tecido biológico de outro. Houve 3 pacientes 
do sexo feminino e 3 do sexo masculino que desenvolveram ISC. Os resultados obtidos nesse artigo não corroboram com a literatura devido não apresentar o isolamento do Staphylococcus aureus que é o agente etiológico mais prevalente ${ }^{17}$

De maneira geral, segue a descrição dos agentes, na tabela 2.

\section{Tabela 2: Microrganismos nos estudos}

\begin{tabular}{|c|c|c|}
\hline ARTIGOS & $\begin{array}{l}\text { MAIOR } \\
\text { PREVALÊNCIA }\end{array}$ & $\begin{array}{l}\text { MENOR } \\
\text { PREVALENCIA }\end{array}$ \\
\hline ARTIGO 1 & $\begin{array}{l}\text { Staphylococcus } \\
\text { aureus }\end{array}$ & $\begin{array}{l}\text { Enterobacter } \\
\text { Cloacae }\end{array}$ \\
\hline ARTIGO 2 & $\begin{array}{l}\text { Pseudomonas } \\
\text { aeruginosa }\end{array}$ & Não relatado \\
\hline ARTIGO 3 & $\begin{array}{l}\text { Staphylococcus } \\
\text { aureus }\end{array}$ & $\begin{array}{l}\text { Klebsiella } e \quad S . \\
\text { epidermids }\end{array}$ \\
\hline ARTIGO 4 & $\begin{array}{l}\text { Staphylococcus } \\
\text { aureus }\end{array}$ & Não relatado \\
\hline ARTIGO 5 & $\begin{array}{l}\text { Staphylococcus } \\
\text { aureus }\end{array}$ & $\begin{array}{l}\text { Klebsiella } \\
\text { pneumonae }\end{array}$ \\
\hline ARTIGO 6 & $\begin{array}{l}\text { Staphylococcus } \\
\text { aureus }\end{array}$ & $\begin{array}{l}\text { S. epidermids e } \\
\text { Enterobacter } \\
\text { cloacae }\end{array}$ \\
\hline
\end{tabular}

Fonte: Dados da pesquisa, 2020

Os agentes etiológicos encontrados no estudo do artigo número sete não foram inseridos na tabela, devido o mesmo não apresentar prevalência maior ou menor do microorganismo, tendo a ocorrência de um Enterobacter cloacae, um Acinetobacter baumannii e um Stenotrophomonas maltophili.

Podemos evidenciar pela tabela, uma ocorrência de $71,42 \%$ de prevalência do Staphylococcus aureus, seja ele resistente à meticilina ou não nas culturas das infecções cirúrgicas em ortopedia, com ênfase em artroplastia de quadril. Em menor prevalência, mas 
não pouco importante, seguem em sequência os agentes Enterobacter cloacae, Klebsiella pneumonae e S.epidermids. O fato de o Staphylococcus aureus ser o mais prevalente nas infecções corrobora com os dados encontrados na literatura acerca do perfil dos microrganismos em infecções ortopédicas.

\section{ARTROPLASTIA DE QUADRIL E FATORES DE RISCO RELACIONADOS}

No segundo artigo avaliado, 185 pacientes não eram fumantes. Em relação à presença de infecções prévias à cirurgia, foi observado que 175 pacientes se apresentavam sem infecção, 29 com infecção urinária, 7 com artrite séptica e 1 com outras infecções. Em relação à cirurgia e o grau de contaminação, foram observadas 57 cirurgias limpas, 2 potencialmente infectadas e 1 infectada. $^{12}$

No estudo de EBIED et al (2016), dos pacientes que se submeteram ao estudo, 22 de 27 pacientes chegaram a apresentar fatores gerais de risco à saúde: $25 \%$ de tabagismo, 18\% hepatopatias, $14 \%$ cardiopatias, $25 \%$ diabetes mellitus, 11 IMC $<35$ e $7 \%$ problemas renais. ${ }^{13}$

Em relação às condições clínicas do paciente, foram diagnosticadas 704 doenças prévias, sendo HAS, hipercolesterolemia e artrose as mais frequentes. O estudo mostrou também que a cada comorbidade, o risco de desenvolvimento de ISC aumenta $0,35 \% .^{14}$

O quinto artigo (PINTO et al, 2015) mostra os diagnósticos obtidos nos pacientes, que foram submetidos a artroplastias, tais como artrose e artrites reumatoides ${ }^{15}$

No trabalho do sexto artigo, 291 eram mulheres e 226 eram homens, com a idade mínima de 18 anos e a máxima de 86; em relação ao IMC, tiveram 7 de baixo peso, 151 eutróficos, 249 sobrepesos e 217 obesos. Em relação à comorbidade dos pacientes, 279 pacientes eram portadores de HAS, 70 de diabetes mellitus, 37 anemia crônica, 34 atrite reumatoide, 18 de algum tipo de neoplasia, 19 de Doença Pulmonar Obstrutiva Crônica 
(DPOC), 14 de Insuficiência Renal Crônica (IRC) e 9 de doenças autoimunes. Além disso, 63 dos pacientes eram fumantes e 13 etilistas. ${ }^{16}$

No artigo de (CUNHA, 2019) das doenças que os pacientes apresentavam, 93 correspondiam a HAS, 18 a Diabetes Mellitus, 9 a cardiopatias, 7 a doenças pulmonares e 7 apresentavam alguma doença autoimune. Em relação ao tabagismo 23 se enquadravam, enquanto 18 eram etilistas. Dos 149 pacientes que realizaram o procedimento cirúrgico, 56 foram encaminhados ao Centro de Tratamento Intensivo (CTI) para cuidados do pósoperatório. Das cirurgias, 68 foram artroplastias total de joelho e 81 compreendiam artroplastia total de quadril, tendo o motivo da cirurgia com coxoartrose de $36,5 \%$, fraturas de $16,2 \%$, gonoartrose de $45,3 \%$ e osteonecrose com $2 \% .^{17}$

Um dos cuidados para se submeter ao procedimento cirúrgico era a cessação do tabagismo. Segundo Hoyos (2012) e Warner (2006), citados por CAVICHIO et al (2014), quando se tem uma alta exposição à nicotina, podem-se gerar alterações fisiológicas, que comprometem a resposta às intervenções e contribuem para que a morbidade no pósoperatório seja aumentada 18, 19, 20.

Nos dados mostrados no estudo, é evidenciado que alguns pacientes eram fumantes e em alguns casos, o tabagismo era acompanhado pelo estilismo, o que pode estar interligado com as taxas de infecções apresentadas em cada artigo, sendo que o tabagismo é colocado como fator de risco para o desenvolvimento da infecção de sítio cirúrgico. O manual da Agência Nacional de Vigilância Sanitária (ANVISA) de medidas de prevenção de infecção relacionadas à assistência à saúde, publicado em 2017, mostra o tabagismo como fator de risco e orienta que a cessação do mesmo seja obrigatória no pré-operatório de cirurgias eletivas, e que isso aconteça por pelo menos 30 dias antes do procedimento. ${ }^{21}$

Em relação às comorbidades, as de maior prevalência relatadas nos artigos compreendem em primeiro lugar a HAS, seguida por Diabetes Mellitus e obesidade. O que 
sustenta um estudo de AGUIAR et al (2012) que fala sobre as infecções e as comorbidades. Segundo esse estudo, a taxa de pacientes com comorbidades era de $34,57 \%$, e que a presença delas tem efeito importante no prognóstico do paciente. ${ }^{22}$

\section{ARTROPLASTIA E ANTIBIOTICOPROFILAXIA}

Em relação à antibioticoprofilaxia destacada no segundo artigo, a prescrição de antibiótico pós-procedimento ocorreu em 97\%, sendo o mais utilizado a cefazolina de $1 \mathrm{~g}$ de 6/6 EV em 73,4\% da amostra. Concluiu-se que a taxa de infecção foi baixa, apesar de haver divergência no protocolo de antibioticoprofilaxia. ${ }^{12}$

Também, no quinto artigo, tem ênfase da cefazolina, mas neste, todos os pacientes na primeira cirurgia receberam um grama de cefazolina, até 30 minutos antes da incisão, e o antibiótico profilático foi mantido por até 24 horas da cirurgia. ${ }^{15}$

Em relação aos antibióticos profiláticos, foram utilizados no período a cefazolina em 53 pacientes, a cefuroxima em 91 e a clindamicina em 3. Já no período do pós-operatório, a cefazolina foi utilizada em 44 pacientes, cefuroxima em 80 , clindamicina em 2 e a teicoplanina em 1. ${ }^{17}$

Em relação à escolha do antibiótico, isso varia de cirurgia, pois deve se considerar o sítio que está sendo operado, se há torniquetes e diagnóstico para a cirurgia ${ }^{21}$

A ANVISA (2017) preconiza a cefazolina como antibiótico de primeira escolha em cirurgias ortopédicas gerais, tendo-se a duração de 24 horas; já em cirurgias ortopédicas como artroplastias primárias, ou revisões de artroplastias, a indicação de primeira escolha seria cefuroxima, sendo que em casos de procedimentos de revisões, deve-se ter a coleta de vários planos para a cultura. Grande parte dos artigos mostra a antibioticoprofilaxia com cefazolina. $^{21}$

\section{PERFIL DAS INFECCÕES APRESENTADAS}


Quando se mostram dados referentes à ISC, é de suma importância que se leve em consideração o tipo de cirurgia, as condições do paciente, a técnica utilizada, a degermação, a antibioticoprofilaxia, entre outros fatores.

Como qualquer outro procedimento, a artroplastia tem suas particularidades. Watne et al (2014) mostram que uma complicação intraoperatória e a duração aumentada de cirurgias foram significativamente comparadas a um risco aumentado de ISC. ${ }^{23}$ Com base nos dados apresentados no trabalho em relação ao perfil dos pacientes, observam-se pacientes pediátricos, pacientes jovens, adultos e idosos. Os estudos apresentaram uma amostra total próxima de 1811 pacientes.

Os artigos apresentados para a discussão neste trabalho foram divididos em relação ao sexo de pacientes que acabaram sendo acometidos por infecções cirúrgicas, e conforme mostrado, o sexo feminino foi o de maior prevalência, mesmo sem justificativas para correlacionar o sexo às infecções.

Os artigos mostram poucos dados acerca da extensão das infecções, algo que deveria ser mais abordado, pois, para que se tenha um protocolo adequado, é de suma importância saber se as infecções que mais acometem os pacientes estão sendo superficiais, profundas ou de órgão/ cavidade, pois isso muda o tratamento do paciente. O artigo 1 (CHAGAS, et al, 2017) mostra que a maioria das infecções foram superficiais, o que difere do artigo 5 de PINTO et al (2015) que mostra que as infecções de órgão/cavidade tiveram maior prevalência. É relatado também neste artigo, que de todos os procedimentos estudados pelos autores, a cirurgia limpa teve mais relevância, seguida de a potencialmente contaminada, o que gera um prognóstico cirúrgico bom, em relação ao desenvolvimento de infecção.

Os critérios para o diagnóstico de infecção superficial, de acordo com a ANVISA (2017) compreendem aquela que ocorreu nos 30 dias após a cirurgia, que atingiu pele e tecido subcutâneo e que apresentou um dos critérios tais como: tecido da incisão superficial obtido 
assepticamente, drenagem purulenta ou cultura positiva de secreção; já a incisional profunda vai ocorrer também nos primeiros 30 dias após o procedimento cirúrgico ou até 90 dias se tiver implante, já envolveu tecidos mais profundos como fáscia e ou músculos, e apresentou pelo menos um dos critérios: drenagem purulenta da incisão profunda, deiscência e cultura positiva ou não realizada. E a infecção de órgão/cavidade também ocorreu nos primeiros 30 dias e ou até 90 dias após procedimento cirúrgico, se tiver implantes, quando atingiu qualquer órgão ou cavidade e que apresentou um dos critérios: cultura positiva, presença de abscesso, que foi identificado na reoperação, exame clínico, anatomopatológico ou de imagem e diagnosticado pelo médico. Ou seja, conforme os critérios, não é uma obrigatoriedade a cultura da secreção/ferida para que se tenha o diagnóstico de infecção de sitio cirúrgico, mas contudo, quando se tem a ciência do agente que está causando a infecção, o tratamento se torna mais correto e mais coerente, sendo assim a infecção não será tratada de forma empírica ${ }^{1}$.

\section{CONCLUSÕES}

Por mais que se tenham vários protocolos e diretrizes acerca das medidas de prevenção de infecções em sítio cirúrgico, indubitavelmente, as comorbidades, tabagismo e técnicas utilizadas têm uma grande relevância e importância no decorrer do tratamento pósoperatório. A prevalência do Staphylococcus aureus nas culturas das infecções tem sido descrita constantemente pela literatura, o que pode levar a estudos mais aprofundados sobre sua associação com as cirurgias ortopédicas. Pode-se evidenciar que a comorbidade é um fator de risco para ISC, contudo tendo a HAS e a DM com maiores prevalências nas

descrições dos casos. É relevante manter os cuidados do pós-operatório para que não desenvolvam infecções cirúrgicas e que assim que puder obter a coleta da cultura e o tratamento seja feito de acordo com o microrganismo encontrado. 


\section{REFERÊNCIAS}

1. BRASIL. Agência Nacional de Vigilância Sanitária. Critérios Diagnósticos de Infecções Relacionadas à Assistência à Saúde/Agência Nacional de Vigilância Sanitária. Brasília: Anvisa, 2017.

2. MARTINS, Tatiana et al. Fatores de risco para infecção do sítio cirúrgico em cirurgias. Texto Contexto Enferm, 2018

3. CARVALHO, Rafael Lima Rodrigues. et al. Incidência e fatores de risco para infecção de sitio cirúrgico em cirurgias gerais. Rev. Latino-Am. Enfermagem 2017;25:e2848 DOI: 10.1590/15188345.1502 .2848

4. SOUZA, Istefânia Soares Borges de Souza; SANTANA. Adriana Cristina; JUNIOR, Geovanne D' Alfonso. A ocorrência de infecção do sítio cirúrgico: um estudo de revisão. Rev Med Minas Gerais 2018.

5. OLIVEIRA, C. b. s. et al. Frequência e perfil de resistência de Klebsiella spp. em um hospital universitário de Natal/RN durante 10 anos • J Bras Patol Med Lab • v. 47 • n. 6 • p. 589-594 • dezembro 2011

6. SANTOS, Neusa de Queroz. A resistência bacteriana no contexto da infecção hospitalar. Texto Contexto Enferm 13(n.esp):64-70 , 2004

7. REIS, Henry Pablo Lopes Campos et al. Avaliação da resistência microbiana em hospitais privados de Fortaleza - Ceará. Rev. Bras. Farm. 94 (1): 83-87, 2013

8. GALIA, CR et al. Atualização em artroplastia total de quadril: uma técnica ainda em desenvolvimento. Revista Brasileira de Ortopedia, v. 52, n. 5, p. 521-527, 2017.

9. BOTELHO, L. L. R.; CUNHA, C. C. A.; MACEDO, M. O método da revisão integrativa nos estudos organizacionais. Gestão E Sociedade · Belo Horizonte, V. 5. No 11 · P. 121-136 · Maio/Ago · ISSN 1980-5756 · www.ges.face.ufmg.br, 2011.

10. ERCOLE, F. F; ALCOFORADO, C. L. G. C.; MELO, L. S. Revisão Integrativa versus Revisão Sistemática. REME Rev Min Enferm, jan/mar; 18(1): 1-260, 2014. 
11. CHAGAS, Mariana de Queiroz Leite et al . Análise das infecções de sítio cirúrgico em pacientes pediátricos após cirurgia ortopédica: um estudo caso-controle. Rev. paul. pediatr., São Paulo , v. 35, n. $1, \quad$ p. 18-24, $\quad 2017$ mar. $\quad$ Disponível em $<\mathrm{http} / / / \mathrm{www}$.scielo.br/scielo.php?script=sci_arttext\&pid=S010305822017000100018\&lng=pt\&nrm=iso>. acessos em 21 set. 2020. https://doi.org/10.1590/19840462/;2017;35;1;00011.

12. DULPIAZ, Jaqueline; RIVA PAGNUSSAT, Lidiane; HAHN, Siomara Regina. Artroplastia de quadril em idosos hospitalizados e o uso de antibioticoprofilaxia. Revista de Epidemiologia e Controle de Infecção, Santa Cruz do Sul, v. 8, n. 4, out. 2018. ISSN 2238-3360. Disponível em: . Acesso em: 17 jan. 2019. doi:https://doi.org/10.17058/reci.v8i4.11870

13. Ebied, A.M., Elseedy, A.I. \& Gamal. O. Um protocolo para artroplastia encenada para salvar a nãp união infectada de fraturas de quadril. $\mathrm{J}$ Orthopaed Traumatol 18, 43-50 (2017). https://doi.org/10.1007/s10195-016-0419-6

14. FRANCO, Lucia Maciel de Castro; ERCOLE, Flavia Falci; MATTIA, Adelaide De. Surgical infection in patients undergoing orthopedic surgery with implant. REV. SOBECC, SÃO PAULO. JUL./SET. 2015; 20(3): 163-170

15. PINTO, Cibele Zdebsky da Silva; ALPENDRE, Francine Taporosky; STIER, Christiane Johnscher Niebel; MAZIERO, Eliane Cristina Sanches; ALENCAR, Paulo Gilberto Cimbalista de; CRUZ, Elaine Drehmer de Almeida. Caracterizacão de artroplastias de quadril e joelho e fatores associados à infecção. rev bras ortop .201 5;50(6):694-699.

16. MIRANDA, Aline Rodrigues de Abreu. Aspectos epidemiológicos das infecções de sítio cirúrgico em cirurgias ortopédicas com implante em um hospital de reabilitação. Dissertação de mestrado. Universidade Federal de Minas Gerais. Belo Horizonte, 2017.

17. CUNHA, Renata Cristina Gonçalves. Incidência e fatores de risco para infecções de sítio cirúrgico ortopédicas com uso de prótese: coorte não concorrente. Dissertação (Mestrado) - Universidade Federal de Minas Gerais, Escola de Enfermagem. Belo Horizonte, 2019.

18. Hoyos A, Southard C, DeCamp MM. Perioperative smoking cessation. Thorac Surg Clin. 2012 Feb;22(1):1-12, v. doi: 10.1016/j.thorsurg.2011.09.006. PMID: 22108683. 
19. WARNER DO. Perioperative abstinence from cigarettes: physiologic and clinical consequences. Anesthesiology. 2006;104(2):356-67

20. CAVICHIO, Barbara Vieira et al . Tempo de cessação do tabagismo para a prevenção de complicações na cicatrização de feridas cirúrgicas. Rev. esc. enferm. USP, São Paulo, v. 48, n. 1, p. 170-176,
fev.
2014
Disponível
em

<http://www.scielo.br/scielo.php?script=sci_arttext\&pid=S0080-

62342014000100170\&lng=pt\&nrm=iso>. $\quad$ acessos $\quad$ em $\quad 21 \quad$ set. 2020 . http://dx.doi.org/10.1590/S0080-623420140000100022.

21. BRASIL. Agência Nacional de Vigilância Sanitária Medidas de Prevenção de Infecção Relacionada à Assistência à Saúde. Brasília: Anvisa, 2017

22. AGUIAR, Ana Paula Lima; PRADO, Patricia Rezende do; OPITZ, Simone Perufo; VASCONCELOS, Suleima Pedroza; FARO, André Ricardo Maia da Costa de. Factors associated with surgical site infections in a hospital in the western Brazilian amazon. Rev. SOBECC, São Paulo. jul./set. 2012; 17(3) 60-70.

23. WATNE, LO, Torbergsen AC, Conroy S, Engedal K, Frihagen F, Hjorthaug GA, Juliebo V, Raeder J, Saltvedt I, Skovlund E, Wyller T B. O efeito de um serviço ortogeriátrico pré e pós-operatório na função cognitiva em pacientes com fratura de quadril: ensaio clínico randomizado (Oslo Orthogeriatric Trial). BMC Med 2014; 12:63. 